\title{
BMJ Open Transition from child to adult health services for young people with cerebral palsy in Ireland: a mixed-methods study protocol
}

\author{
Jennifer M Ryan, ${ }^{1}$ Jennifer Fortune (D) , ${ }^{1}$ Aisling Walsh (D) , ${ }^{1}$ Meriel Norris, ${ }^{2} \mathrm{C}$ Kerr, ${ }^{3}$ \\ Owen Hensey, ${ }^{4}$ Thilo Kroll, ${ }^{5}$ Grace Lavelle, ${ }^{6}$ Mary Owens, ${ }^{7}$ M Byrne, ${ }^{8}$ \\ Michael Walsh ${ }^{9}$
}

To cite: Ryan JM, Fortune J, Walsh A, et al. Transition from child to adult health services for young people with cerebral palsy in Ireland: a mixed-methods study protocol. BMJ Open 2020;10:e041425. doi:10.1136/ bmjopen-2020-041425

- Prepublication history and additional material for this paper is available online. To view these files, please visit the journal online (http://dx.doi.org/10. 1136/bmjopen-2020-041425)

Received 09 June 2020 Revised 12 November 2020 Accepted 27 November 2020

Check for updates

(C) Author(s) (or their employer(s)) 2020. Re-use permitted under CC BY-NC. No commercial re-use. See rights and permissions. Published by BMJ.

For numbered affiliations see end of article.

Correspondence to Jennifer M Ryan; jenniferryan@rcsi.ie

\section{ABSTRACT}

Introduction The transition from child to adult health services is a challenging and complex process for young people with cerebral palsy (CP). Poorly managed transition is associated with deterioration in health, increased hospitalisations and reduced quality of life. While international research identifies key practices that can improve the experience and outcomes of transition, there is a paucity of data in the Irish context. This research study aims to gain an insight into the experience of transition for young people with CP in Ireland.

Methods and analysis A convergent parallel mixedmethods design will be used to collect, analyse and interpret quantitative and qualitative data. Participants will be young people aged 16-22 years with $\mathrm{CP}$, their parent(s)/carer(s) and service providers. Quantitative and qualitative data will be collected through questionnaires and interviews, respectively. Quantitative data will be reported using descriptive statistics. Where sufficient data are collected, we will examine associations between the experience of transition practices and sociodemographic and CP-related factors, respectively, using appropriate regression models. Associations between service provider characteristics and provision of key transition practices may also be explored using appropriate regression models. Qualitative data will be analysed using the Framework Method. A coding matrix based on key transitional practices identified from the literature will be used to identify convergence and divergence across study components at the integration stage.

Ethics and dissemination The study has been approved by the RCSI University of Medicine and Health Sciences Research Ethics Committee (REC201911010). Results will be presented to non-academic stakeholders through a variety of knowledge translation activities. Results will be published in open access, peer-reviewed journals and presented at national and international scientific conferences.

\section{INTRODUCTION}

Cerebral palsy (CP) is one of the most prevalent disabling conditions among children worldwide. ${ }^{1} \mathrm{CP}$ is an umbrella term used to describe a group of lifelong conditions that
Strengths and limitations of this study

- This study will provide a comprehensive understanding of the experiences of transition among young people with cerebral palsy in Ireland.

- This study uses a mixed-methods design to integrate quantitative and qualitative data from multiple stakeholders.

- This study involves young people with cerebral palsy, their parent(s)/carer(s) and service providers throughout the study to ensure relevance, responsiveness to needs and translation of findings to practice.

- Transferability to other health and social care systems will be limited as all data will be generated in Ireland.

- Findings from quantitative data are at risk of selection bias as convenience sampling will be used and participation is voluntary.

affect movement and posture causing activity limitations, which result from disturbances to the developing fetal or infant brain. ${ }^{1}$ Many people with CP also experience associated impairments such as sensory, communication and cognitive impairments. ${ }^{1}$ The type and severity of impairment vary considerably among people with CP. The majority of children with CP will survive into adulthood, with many having a life expectancy similar to that of the general population. ${ }^{2}$ Continual improvements in longevity have resulted in a transitional care period between child and adult health services for young people with CP. Transition is defined as 'the purposeful planned process that addresses the medical, psychosocial, educational and vocational needs of adolescents and young adults with chronic medical and physical conditions as they move from child-centred to adultorientated healthcare systems'. ${ }^{3}$ Despite the 
importance of transition for supporting the long-term health and well-being of people with chronic conditions, international research indicates that this process is poorly managed for young people with $\mathrm{CP}^{4-7}$ Poor management of transition is associated with deterioration in health, increased hospitalisations and reduced quality of life among young people with long-term conditions. ${ }^{8-11}$ For young people with cerebral palsy, transition from child to adult health services coincides with a decrease in visits to specialist and coordinated services, difficulties accessing clinical care $^{12}$ and an increase in unmet health needs. ${ }^{5}$ This is concerning given a growing body of evidence shows that adults with $\mathrm{CP}$ experience an increasing number of physical and mental health conditions with age, such as cardiovascular disease, arthritis, depression and anxiety. ${ }^{13} 14$ These conditions often co-occur with worsening physical functioning, pain, fatigue and joint deformities ${ }^{15}$ due to musculoskeletal progression, or the comorbidities associated with CP. ${ }^{16}$ Poor management of transition from child to adult health services may therefore contribute to the development of chronic conditions among adults with CP.

A recent review of the quality of care provided to young people with CP in the UK highlighted that young people have difficulty in navigating adult services, a lack of multidisciplinary teams and leads for adult neurodisability care, and that young people increasingly rely on general practitioners (GPs), after discharge from child services. ${ }^{17}$ Further challenges to implementing successful transition for young people in the UK include differences between the funding and organisational structure of child and adult services, lack of service level coordination, lack of involvement of GPs in transition planning ${ }^{18}$ and inadequate training of adult health professionals in paediatric conditions. ${ }^{19}{ }^{20}$ However, a recent programme of research also identifies key practices associated with improved outcomes including a level of parental involvement that is deemed appropriate by both the young person and parent, promotion of health self-efficacy, meeting the adult team before transfer and incorporation of developmentally appropriate healthcare throughout the transition process. ${ }^{18}$

\section{Transition in an Irish context}

The National Policy and Strategy for the Provision of Neuro-Rehabilitation Services in Ireland and the Integrated Care Programme for Children emphasise the need for appropriate planning and clear protocols to support transition to adult services for young people in Ireland. ${ }^{21}{ }^{22}$ However, there is no research examining transition for young people with CP in Ireland. Two studies have examined the experience of transition in an Irish context by surveying parents of young people with cystic fibrosis ${ }^{23}$ and consultant psychiatrists working in mental health services ${ }^{24}$ respectively. Both studies identified a lack of standardised national practice, lack of written transition plans, lack of interaction between child and adult services and lack of information on self-management and signposting to alternative supports. While this research suggests that transition is poorly managed within the Irish healthcare system, examination of whether these findings are applicable to young people with $\mathrm{CP}$ warrants investigation. Moreover, existing evidence regarding transition in the Irish context focuses on the views of parents and service providers. The voice of young people with disabilities or long-term conditions has not been engaged with to date.

In order to facilitate implementation of successful transition for young people with CP in Ireland, we need to first assess the gaps in the current management of transition, and second explore the experiences of transition from the multiple perspectives of young people with CP, their families and health professionals on how to implement successful transition in an Irish context.

\section{Aim}

The aim of this study is to examine how transition care is provided to and experienced by young people with CP in Ireland.

The specific objectives are as follows:

1. To assess the experience of key transition practices among young people with CP and their parent(s) / carer(s) in the Irish healthcare system.

2. To assess the provision of key transition practices to young people with $\mathrm{CP}$, from the perspectives of disability service providers in the Irish healthcare system.

3. To explore the perspectives of young people with CP and their parent(s)/carers(s) on how to implement transition in the Irish healthcare system.

4. To explore the perspectives of service providers on transition for young people with CP.

\section{METHODS AND ANALYSIS}

We will use a convergent parallel mixed-methods design to address the overall aim of this study. Quantitative and qualitative data will be collected and integrated at the interpretation stage. This study will be conducted between January 2020 and December 2021. Figure 1 illustrates the planned timeline. Recruitment is ongoing.

\section{Framework of key transition practices}

The following framework of key transition practices was informed by a recent research programme conducted in the UK, ${ }^{182}$ the United Kingdom's National Institute for Health and Care Excellence guideline on transition, ${ }^{26}$ and relevant Irish policy documents ${ }^{21222728}$ as practices that improve the experience and outcomes of transition from child to adult services (online supplemental appendix 1 ). The framework was also informed by discussions with members of the Project Management and Study Steering Groups, which includes health professionals, young people with CP and parents. This framework will be used to assess gaps in the current management of transition and to explore enablers, barriers and supports required to implement successful transition in the Irish healthcare system, for young people with CP. 


\begin{tabular}{|c|c|c|}
\hline & & Month \\
\hline Phase & Deliverable & 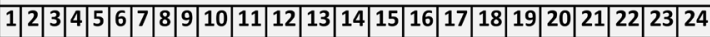 \\
\hline \multirow[t]{2}{*}{ Study preparation } & Develop and pilot study documentation & \\
\hline & Obtain ethical approval & \\
\hline \multirow[t]{3}{*}{ Data collection and analysis } & Quantitative data & \\
\hline & Qualitative data & \\
\hline & Data integration & \\
\hline \multirow{2}{*}{ Dissemination } & Prepare and submit manuscript & \\
\hline & Produce dissemination materials & \\
\hline
\end{tabular}

Figure 1 Gantt chart outlining study timeline.

1. A named worker, known to the young person, who oversees, coordinates or delivers transition support, acts as the link between the young person and the various practitioners involved in their care including their GP. This person may not be formally allocated and may not be a health provider but should be in close contact with health services.

2. Involvement of parent(s)/carer(s) in the young person's care at a level that is deemed appropriate by both the young person and parent $(\mathrm{s}) / \operatorname{carer}(\mathrm{s})$.

3. Child and adult services provide young people and families with information that describes the transition process and the support available before and after transfer. The information should specifically mention health services, which encompasses services that have a direct impact on people's physical health, mental health and social well-being. This information should be provided early enough to allow young people time to reflect and discuss with parent(s)/carer(s) or health professionals and be in an accessible format. Where there is no adult service for a young person to transfer to, information about known and trusted voluntary organisations who could provide support should be provided to the young person.

4. Promotion of health self-efficacy (ie, actively helping young people to feel confident in managing their condition), where health encompasses complete physical, mental and social well-being, including provision of information to the young person about their condition and encouragement to take responsibility for their health.

5. Promotion of opportunities for self-management, where the individual is directly involved in planning and decision-making around their needs and takes responsibility for maintaining optimal physical, mental and social well-being.

6. A health professional from the relevant adult services or primary care meets the young person before they transfer from child services

7. A senior manager with responsibility for implementing transition strategies and policies, including facilitating good working relationships between child and adult services, ensuring appropriate materials are available, and monitoring that the person has a suitable appointment in adult services. This person may not be known to the young person
8. Where there is no adult service for a young person to transfer to, a detailed discharge letter is sent to the young person's GP.

9. Formal training, relevant to health condition, in wider life skills-education, gaining employment, finances, housing, social relationships, sexual health, mental health. The health service may not provide such training but during consultations, staff should inquire about such matters and make referrals to other agencies as needed.

\section{Patient and public involvement statement}

A Study Steering Group of young people with CP, parent(s)/carer(s) and service providers will work closely with the Project Management Group and advise on all steps in the research processes. This will include developing study documentation such as information sheets, developing and piloting questionnaires, developing and piloting topic guides and interpreting findings. The Study Steering Group has informed the development of the framework of key transition practices. The group will also contribute to the development of materials to share findings with young people, families and service providers, such as an information leaflet and infographic. Further, based on the findings, the group will support the Project Management Group to identify and develop resources to facilitate successful transition for young people with CP in Ireland.

\section{Participants}

Participants will be young people with CP aged 16-22 years, their parent(s) or carer(s), and individuals who provide health services to young people with $\mathrm{CP}$ who are residing or working in Ireland. We will include people with CP of all severities of physical impairment.

\section{Recruitment}

Participants will be recruited from across Ireland via disability service providers, special education needs schools, universities, general practices, professional organisations and organisations for people with disabilities and through media, social media and word of mouth. Advertisements and participant information leaflets will include the URL to access an online version of the questionnaire and contact details for the research team, so that participants may request further information or the questionnaire in alternative formats. 
Young people, their parents or carers and service providers who complete the questionnaire will be asked to contact a member of the research team if they would like to participate in an interview. We will develop a sampling frame and purposively sample young people, parents or carers and service providers for interview based on characteristics that may affect the experience or provision of transition. For young people, these may include age, gross motor function, intellectual disability and geographical location. For service providers, these may include the professional role of the individual, sector and geographical location.

\section{Quantitative data collection}

We will aim to collect quantitative data from up to 100 service users (ie, young people, parent(s), carer(s)) and service providers via a questionnaire. Participants may complete the questionnaire online, in person, by post or by telephone or video-conference. Young people may obtain support from a parent, family-member or carer to complete the questionnaire. To maximise inclusivity, we will make the questionnaire accessible to people with varying impairments including mild-to-moderate intellectual disability, for example by providing the survey in alternative formats and using various enhancements such as visual aids, simple words, clear questions and story technique. Where it is not possible to provide sufficient adaptations to obtain the young person's perspectives, a parent or carer may complete the questionnaire on their behalf. The questionnaire was developed in collaboration with, and piloted on young people, parents and service providers prior to use.

The following data will be collected from young people with CP:

1. Sociodemographic and condition-specific data.

2. Current and previous service use.

3. Experience of key transition practices.

4. Unmet health needs using an unmet needs questionnaire. $^{5}$

The following data will be collected from service providers:

1. Service-related data.

2. Predominant transition model.

3. Provision of key transition practices.

\section{Qualitative data collection}

Face-to-face, telephone or videoconference in-depth interviews will be conducted with service users and service providers. Approximately 25 service users and 20 service providers will participate in interviews. Service user interviews may be conducted individually with the young person or jointly with young people and their parent(s) or carer(s). If the young person is under 18 years, they must be interviewed with a parent or guardian present. Adaptations will be made within the interview to allow for inclusion of people with mild-to-moderate intellectual disability, communication impairment, visual impairment, hearing difficulty or other impairments. These may include providing them with the topic guide in lay language in advance, using alternative systems of communication and providing pictorial memory aids. Individual interviews with parent(s)/carer(s) will be facilitated where it is not possible to provide sufficient adaptations to obtain the young person's perspectives. Interviews will be conducted at the participant's home, workplace or other suitable and convenient venue.

We will use the framework of key transition practices to develop the topic guide for interviews. However, we will also explore participants' perspectives of transition more broadly. Separate topic guides for service users and service providers will be developed in collaboration with and piloted on young people, parents and service providers.

Examples of topics to be explored during interviews with service users will include (1) experience of key transition practices; (2) if they believe these practices improved the experience of transition or in the case where practices were not experienced if these practices may have improved their experience; (3) how these practices should be implemented, for example, through structured programmes, written information, verbal information and (4) other supports that improved or would improve their experience of transition. Examples of topics to be explored during interviews with service providers will include (1) perceptions of successful and unsuccessful transition; (2) barriers and enablers to implementing key transition practices including organisational structures, processes and relationships; (3) readiness for implementation and (4) supports required to implement successful transition including the content and format of resources to facilitate implementation.

\section{Analysis}

Descriptive statistics will be used to report quantitative data such as sociodemographic data and experience of key transition practices. Where sufficient data are collected, we will examine associations between the experience of transition practices and sociodemographic and CP-related factors (eg, Gross Motor Function Classification System (GMFCS) level), respectively, using appropriate regression models. Associations between service provider characteristics and provision of key transition practices may also be explored using appropriate regression models where sufficient data are available.

Interviews will be analysed using the Framework Method, which allows for both deductive and inductive analysis of data. The Framework Method is appropriate for this study as we have predefined topics that we wish to explore but are also open to the emergence of additional themes. The Framework Method involves five iterative stages: familiarisation, thematic framework identification, labelling, charting and mapping and interpretation. ${ }^{29} \mathrm{~A}$ sample of transcripts will be read by two members of the research team until familiarity with the data is established. They will independently develop provisional codes before agreeing on an initial framework through discussion. The initial framework 
developed will be applied to all transcripts and will be iteratively refined through our analytic process if new codes are identified. Analysis will be undertaken by one researcher. A proportion of coded transcripts will be checked by a second researcher. We will explore if demographic and CP-related factors (eg, GMFCS level) influence participants' perspectives of transition. Data will be arranged into charts that summarise themes, issues and individual responses. Finally, members of the Project Management Group will discuss emerging categories and themes collaboratively. Preliminary results and interpretations will also be shared with the study steering group who will have the opportunity to reflect and input on the findings. Strategies to enhance the trustworthiness of the findings, such as negative case analysis, peer-debriefing and reflexivity, will be used.

\section{Integration}

Integration of quantitative and qualitative data will be carried out at the interpretation stage of the research. ${ }^{30}$ We will use the key transition practices as a framework for developing themes and meta-themes. We will search for data related to each theme in questionnaires and interviews. We will also identify any additional emerging themes from questionnaires and interviews. We will use a convergence coding matrix to integrate themes and to develop meta-themes. ${ }^{31}$ This will involve grouping themes according to similar concepts and interpreting them to generate meta-themes. We will search for agreement and disagreement between studies according to meta-themes. Agreement and disagreement will be defined as convergence (ie, findings agree directly), complementarity (ie, findings offer complimentary information), dissonance (ie, findings seemingly contradict each other) or silence (ie, themes arise in quantitative data or qualitative data but not both). Integration will be supported by discussions with the Project Management Group and Study Steering Group.

\section{Data management}

A data management plan will be created prior to the start of data collection. All data will be stored securely. All participants will be assigned an ID number, which will be recorded on study documentation. Anonymous data entered electronically via Online Surveys will be downloaded securely into a study database. Data collected on paper forms will be entered into the study database and paper forms will be stored securely in a locked cabinet. Data entry and validation will be a continuous process. Audio files will be downloaded to an encrypted laptop on completion of interviews before being transferred to secure servers. Audiofiles will be transcribed verbatim by a professional transcription service and imported into qualitative data management software (Nvivo V.12: QSR International). Pseudonyms will be used to report participant quotes with consent. Identifying details will be removed to preserve participant anonymity.

\section{Project management}

The Project Management Group, consisting of the Chief Investigator (JR), co-applicants for the grant and postdoctoral researcher will be responsible for delivering all objectives.

\section{ETHICS AND DISSEMINATION}

This study has been approved by the RCSI University of Medicine and Health Sciences Research Ethics Committee (REC201911010). All participants will provide informed consent prior to data collection. For young people aged 16-18 years, a parent/guardian will also provide consent for the young person to participate. Examples of the participant information sheet (online supplemental material 1) and consent form (online supplemental material 2) are available in the online supplementary material. Information for potential participants is also shared on the study website https://www.ignitionstudy.com. We will use findings from this research, and discussion with the Study Steering Group, to identify resources that will facilitate successful transition from child to adult services for young people with $\mathrm{CP}$ in Ireland. A detailed dissemination plan will be developed in the early phases of the study. We will use a variety of knowledge translation activities to ensure that the outputs generated from this research are accessible to non-academic stakeholders including young people with $\mathrm{CP}$, families and service providers. We will work closely with the Study Steering Group to identify and develop materials to share findings, such as an information leaflet and infographic summarising the findings and a seminar for young people, families and service providers. Results will be published in open access peerreviewed journals and presented at national and international scientific conferences.

\section{Author affiliations}

${ }^{1}$ Department of Public Health and Epidemiology, RCSI University of Medicine and Health Sciences, Dublin, Ireland

${ }^{2}$ College of Health, Medicine and Life Sciences, Brunel University London, Uxbridge, UK

${ }^{3}$ School of Nursing and Midwifery, Queen's University Belfast, Belfast, UK

${ }^{4}$ Medical Department, Central Remedial Clinic, Dublin, Ireland

${ }^{5}$ School of Nursing, Midwifery and Health Systems, University College Dublin, Dublin, UK

${ }^{6}$ Institute of Psychiatry, Psychology \& Neuroscience, King's College London, London, UK

${ }^{7}$ Physiotherapy Department, Central Remedial Clinic, Dublin, Ireland

${ }^{8}$ National Disability Children \& Families Team, Social Care Division, Health Service Executive, Dublin, Ireland

${ }^{9}$ Office of the Chief Clinical Officer, Health Service Executive, Dublin, Ireland

Contributors All authors listed meet the International Committee of Medical Journal Editors criteria for authorship. JR and MW conceived the study, MN, $\mathrm{OH}$, MO, TK, CK, AW, GL and MB designed the study. JR will lead the running of the study. JF will lead the collection, management and analysis of the data. MN and AW will lead the qualitative analysis. JR will lead the statistical analysis. All authors have read and approved the final manuscript.

Funding This work was supported by the Health Research Board, grant number APA-2019-004. The study sponsor is RCSI University of Medicine and Health Sciences.

Competing interests None declared. 
Patient consent for publication Not required.

Provenance and peer review Not commissioned; externally peer reviewed.

Supplemental material This content has been supplied by the author(s). It has not been vetted by BMJ Publishing Group Limited (BMJ) and may not have been peer-reviewed. Any opinions or recommendations discussed are solely those of the author(s) and are not endorsed by BMJ. BMJ disclaims all liability and responsibility arising from any reliance placed on the content. Where the content includes any translated material, BMJ does not warrant the accuracy and reliability of the translations (including but not limited to local regulations, clinical guidelines, terminology, drug names and drug dosages), and is not responsible for any error and/or omissions arising from translation and adaptation or otherwise.

Open access This is an open access article distributed in accordance with the Creative Commons Attribution Non Commercial (CC BY-NC 4.0) license, which permits others to distribute, remix, adapt, build upon this work non-commercially, and license their derivative works on different terms, provided the original work is properly cited, appropriate credit is given, any changes made indicated, and the use is non-commercial. See: http://creativecommons.org/licenses/by-nc/4.0/.

\section{ORCID iDs}

Jennifer Fortune http://orcid.org/0000-0001-8971-1236

Aisling Walsh http://orcid.org/0000-0002-5312-5101

\section{REFERENCES}

1 Rosenbaum P, Paneth N, Leviton A, et al. A report: the definition and classification of cerebral palsy April 2006. Dev Med Child Neurol Suppl 2007;109:8-14.

2 Blair E, Langdon K, Mclntyre S, et al. Survival and mortality in cerebral palsy: observations to the sixth decade from a data linkage study of a total population register and national death index. BMC Neurol 2019;19:111.

3 Blum RW, Garell D, Hodgman $\mathrm{CH}$, et al. Transition from childcentered to adult health-care systems for adolescents with chronic conditions. A position paper of the Society for adolescent medicine. $J$ Adolesc Health 1993;14:570-6.

4 Colver A, Pearse R, Watson RM, et al. How well do services for young people with long term conditions deliver features proposed to improve transition? BMC Health Serv Res 2018;18:337.

5 Solanke F, Colver A, McConachie H, et al. Are the health needs of young people with cerebral palsy met during transition from child to adult health care? Child Care Health Dev 2018;44:355-63.

6 Hilberink SR, Roebroeck ME, Nieuwstraten W, et al. Health issues in young adults with cerebral palsy: towards a life-span perspective. $J$ Rehabil Med 2007;39:605-11.

7 Scal P, Ireland M. Addressing transition to adult health care for adolescents with special health care needs. Pediatrics 2005;115:1607-12.

8 Cramm JM, Strating MMH, Sonneveld HM, et al. The longitudinal relationship between satisfaction with transitional care and social and emotional quality of life among chronically ill adolescents. App Res Qual Life 2013;8:481-91.

9 Lotstein DS, Seid M, Klingensmith G, et al. Transition from pediatric to adult care for youth diagnosed with type 1 diabetes in adolescence. Pediatrics 2013;131:e1062-70.

10 Nakhla M, Daneman D, To T, et al. Transition to adult care for youths with diabetes mellitus: findings from a universal health care system. Pediatrics 2009;124:e1134-41.

11 Yeung E, Kay J, Roosevelt GE, et al. Lapse of care as a predictor for morbidity in adults with congenital heart disease. Int $J$ Cardiol 2008;125:62-5.
12 Roquet M, Garlantezec R, Remy-Neris O, et al. From childhood to adulthood: health care use in individuals with cerebral palsy. Dev Med Child Neurol 2018;60:1271-7.

13 Peterson MD, Ryan JM, Hurvitz EA, et al. Chronic conditions in adults with cerebral palsy. JAMA 2015;314:2303-5.

14 Smith KJ, Peterson MD, O'Connell NE, et al. Risk of depression and anxiety in adults with cerebral palsy. JAMA Neurol 2019;76:294-300.

15 Opheim A, Jahnsen R, Olsson E, et al. Walking function, pain, and fatigue in adults with cerebral palsy: a 7-year follow-up study. Dev Med Child Neurol 2009;51:381-8.

16 Bromham N, Dworzynski K, Eunson P, et al. Cerebral palsy in adults: summary of NICE guidance. BMJ 2019;364:I806.

17 NCEPOD. The National confidential enquiry into patient outcome and death. each and every need. London; 2018.

18 Colver A, Rapley T, Parr JR, et al. Facilitating transition of young people with long-term health conditions from children's to adults healthcare services - implications of a 5-year research programme. Clin Med 2020;20:74-80.

19 Tuffrey C, Pearce A. Transition from paediatric to adult medical services for young people with chronic neurological problems. J Neurol Neurosurg Psychiatry 2003;74:1011-3.

20 Kolehmainen N, McCafferty S, Maniatopoulos G, et al. What constitutes successful commissioning of transition from children's to adults' services for young people with long-term conditions and what are the challenges? An interview study. BMJ Paediatr Open 2017;1:e000085.

21 Department of Health. National policy and strategy for the provision of Neuro-Rehabilitation services in Ireland 2011-2015, 2011.

22 National Clinical Programme for Paediatrics and Neonatology. A national model of care for paediatric healthcare services in Ireland. Chapter 8: Integrated care. Ireland: HSE https://www.hse.ie/eng/ services/publications/clinical-strategy-and-programmes/paediatricneurodisabilty.pdf

23 Coyne I, Malone $\mathrm{H}$, Chubb E, et al. Transition from paediatric to adult healthcare for young people with cystic fibrosis: parents' information needs. J Child Health Care 2018;22:1367493518768448:646-57.

24 McNamara N, McNicholas F, Ford T, et al. Transition from child and adolescent to adult mental health services in the Republic of ireland: an investigation of process and operational practice. Early Interv Psychiatry 2014;8:291-7.

25 Colver AF, Merrick H, Deverill M, et al. Study protocol: longitudinal study of the transition of young people with complex health needs from child to adult health services. BMC Public Health 2013;13:675

26 NICE. National Institute for Health and Care Excellence. Transition from children's to adults' services for young people using health or social care services, 2016. Available: https://www.nice.org.uk/ guidance/ng43

27 National clinical programme for paediatrics and neonatology. A national model of care for paediatric healthcare services in Ireland. Chapter 33: Neurodisability. Ireland: HSE https://www.hse.ie/eng/ services/publications/clinical-strategy-and-programmes/paediatricneurodisabilty.pdf

28 National Strategy \& Policy for the Provision of Neuro-Rehabilitation Services in Ireland From Theory to Action Implementation Framework 2019-2021: Health Service Executive 2019.

29 Ritchie JL J. Qualitative research practice: a guide for social science students and researchers. London: Sage Publishers Inc, 2009.

30 O'Cathain A, Murphy E, Nicholl J. Three techniques for integrating data in mixed methods studies. BMJ 2010;341:c4587.

31 Farmer T, Robinson K, Elliott SJ, et al. Developing and implementing a triangulation protocol for qualitative health research. Qual Health Res 2006;16:377-94. 